\title{
ARTICLE
}

\section{Fast Neutron and Gamma induced Radiation Dose Monitoring during Nano-particle Production by Proton beam Irradiation}

\author{
H. J. KIM ${ }^{1 *}$, Wan Kim ${ }^{1}$, Sangjun KANG ${ }^{2}$, Kyeryung $\mathrm{KIM}^{3}$, Sangpil YUN ${ }^{3}$ \\ ${ }^{I}$ Department of Physics, Kyungpook National University, Daegu, Korea \\ ${ }^{2}$ School of Liberal Art, Semyung University, Jechon, Korea \\ ${ }^{3}$ Atomic Energy Research Institute, P.O. Box 105, Yuseong, Daejeon, Korea
}

\begin{abstract}
We report a fast neutron and gamma induced radiation dose and flux monitoring study during the nano-particle production at the proton beam irradiation facility. The monitoring results will be used for understanding of the activation of irradiated samples and the effect to the nano-particle production. A cylindrical shape of BC523A liquid scintillator was used for the fast neutron and gamma monitoring purpose. A neutron tagger module was used for the signal digitization and it has a function of pulse shape discrimination capability by using a charge comparison method. Samples for the nano-particle production were irradiated with $45-\mathrm{MeV}$ proton beam from the MC-50 cyclotron at the Korea Institute of Radiological and Medical Sciences. The fast neutron detector was located just behind of the sample. Proton beam current from $20 \mathrm{nA}$ to $500 \mathrm{nA}$ was used for the test. The measured neutron and gamma flux, energy spectra, and neutron and gamma induced radiation dose in the liquid scintillator were evaluated and compared with different sample setup. This study showed that the developed detection system is useful for the absorbed dose and flux monitoring of neutron and gamma in the proton beam line.
\end{abstract}

KEYWORDS: nano-particle, neutron monitoring, liquid scintillator, pulse shape discrimination, dose, flux

\section{Introduction}

The identification of the fast neutron and energy spectrum measurement with a liquid scintillator is a very promising technique. ${ }^{1)}$ In the following applications, measuring the fast neutron flux and energy spectrum are important ${ }^{2-3)}$ :

1) Neutron flux monitoring at a high flux proton or hadron environment,

2) Fast-neutron beam line,

3) Detector irradiation study for high-energy physics and nuclear physics in a high-radiation-level environment,

4) Environmental neutron monitoring,

5) Fast neutron monitoring outside of a nuclear reactor,

6) International safeguards and national security,

7) Fast neutron flux measurement at underground laboratory.

Recently, nano-particle production by the proton beam irradiation was developed and $\mathrm{Au}$ and $\mathrm{Ag}$ nano-particles were successfully produced. ${ }^{4-5)}$ It is necessary to study the activation of produced nano-particle samples and the effect to the nano-particle production by the neutrons and gammas which were produced during the proton beam irradiation.

The purpose of this study is to measure the flux, energy spectrum and absorbed dose by the neutron and gamma using a BC523A neutron detector when samples were located at the proton irradiation facility for the nano-particle production.

\section{Experimental setup}

*Corresponding Author, E-mail:hongjoo@knu.ac.kr

\section{Neutron Tagger Module}

A portable neutron tagger module (NTM) by using digital pulse shape discrimination (PSD) method was developed for the neutron and gamma separation in real time. $\left.{ }^{6}\right)$ A digital charge comparison (DCC) method ${ }^{7}$ was implemented, which uses total to tail integration charge for comparison. Since a Field Programmable Gate Array (FPGA) digital signal processor is used for the DCC logic implementation, the other PSD logic can be also implemented without modifying hardware. The FPGA also drives TCP/IP interface to communicate with the outside world by a 10T/100T Ethernet. The sustained maximum throughput of this interface was measured to be $3 \mathrm{Mbytes} / \mathrm{s}$. The physical dimension of the NTM is $6 \mathrm{~cm} \times 13 \mathrm{~cm}$ without a power supply and case so that it is portable and it can be used in the field or beam line science.

By the software, following settings are possible.

1) Total pulse width for charge integration : 20 to 1,270 ns.

2) Delay of peak to tail start time : 0 to $1000 \mathrm{~ns}$.

3) Threshold setting : $10 \mathrm{mV}-1,000 \mathrm{mV}$.

4) Internal gain : 0.7 to 10 .

5) Positive or negative polarity.

6) The tail to total charge ratio for neutron tagging.

The output consists of total charge, tail charge, neutron flag and counter information. It can trigger maximum $500 \mathrm{kHz}$ with 200 ns pulse width setting.

\section{The BC523A Liquid Scintillator and Calibration}

We performed a test with BC523A liquid scintillator (produced by Bicron Co.) which contains $4.41 \%$ of ${ }^{10} \mathrm{~B}$ with the cylindrical shape of $\Phi 50 \times 50 \mathrm{~mm}$. It can 
be utilized for the fast or thermal neutron tagging. The NTM module was tested with the BC523A detector since it has a good PSD capability for the fast neutron tagging. A 2" H1161 PMT (produced by Hamamatsu) was attached directly to the window of the detector and the high voltage of $-1400 \mathrm{~V}$ was applied to the PMT. The PMT output was directly fed into the NTM module

By software, $40 \mathrm{mV}$ of threshold was applied to trigger an event by a self-trigger algorithm, total pulse width for the charge integration was set to $200 \mathrm{~ns}$, delay from peak to tail start time was set to $20 \mathrm{~ns}$ and internal gain was set to 1.2. These parameters were chosen for the optimum $\mathrm{n} / \gamma$ separation. Total integration charge, tail integration charge, neutron tagging flag and counter information were recorded into a laptop computer through TCP/IP connection and the recorded data was analyzed with a $\mathrm{C}^{++}$data analysis program based on ROOT framework. ${ }^{8)}$ A $1 \mu \mathrm{Ci}{ }^{137} \mathrm{Cs}$ radioactive source was used for the electron equivalent energy (Eee) calibration of the BC501A detector and a 1 $\mu \mathrm{Ci}{ }^{252} \mathrm{Cf}$ neutron fission source was used for optimization study of the neutron and gamma separation.

\section{MC-50 Proton Beam Line}

The $45-\mathrm{MeV}$ proton beam test facility at the MC-50 cyclotron of KIRAMS (Korea Institute of Radiological \& Medical Sciences) was established by the PEFP (Proton beam Engineering Frontier Project) of the Korea Atomic Energy Research Institute (KAERI). This facility is used for pilot studies of the PEFP, especially, for studies using a very low proton beam flux from $0.2 \mathrm{nA}$ to $20 \mathrm{nA}$. $^{9}$ The beam line is composed of a collimator, a Faraday cup, a beam profile monitor, an aluminum exit window for an external beam, a phosphor screen, measurement system, etc. ${ }^{9)}$

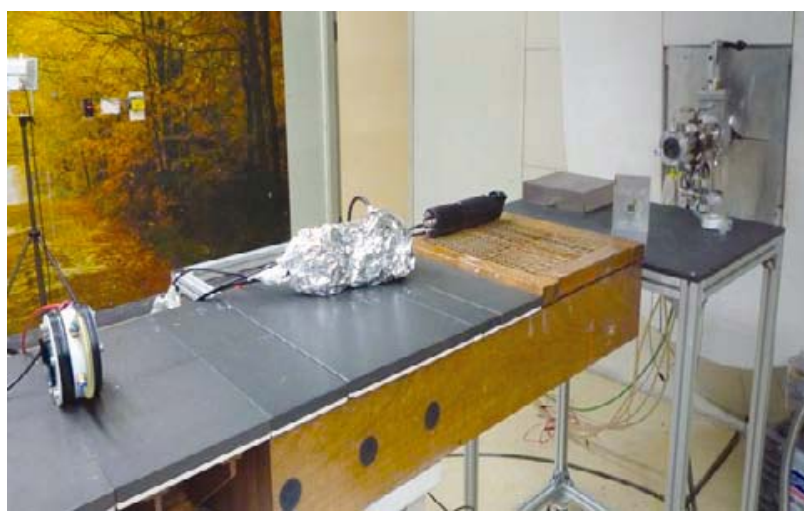

Fig. 1 A photograph of the PEFP beam line with the neutron detector (close to the beam exit) and ion chambers.

The 45.0-MeV incoming proton beam passes through a $0.2 \mathrm{~cm}$ thick aluminum window capping the beam pipe with $40 \mathrm{~cm}$ air and loses energy down to $38 \mathrm{MeV}$. Proton currents from $20 \mathrm{nA}$ to $500 \mathrm{nA}$ were used for this study. Figure 1 shows an exit window in the proton beam line with a BC523A liquid scintillator attached to a PMT. The beam profile at the exit window was measured with double-layer
GafChromic MD-55 system (ISP Technology Inc).

\section{Results}

\section{Calibration and Optimization of $n / \gamma$ Separation}

A ${ }^{137} \mathrm{Cs}$ radioactive source was used for the electron equivalent energy (Eee) calibration of the BC501A detector. The peak energy of the Compton edge irradiated with the $662 \mathrm{keV} \gamma$-ray radioactive source was estimated with the help of Monte Carlo simulation, and it was used for the energy calibration. We obtained a Eee calibration constant of $0.38 \mathrm{keV} /$ Channel.

A ${ }^{252} \mathrm{Cf}$ neutron fission source was used for the study of neutron and gamma separation. High voltage, threshold, and total pulse width and delay of tail start time were optimized for the best performance of neutron and gamma separation. With above optimization process, we set the PMT high voltage of $-1400 \mathrm{~V}$, threshold of $40 \mathrm{mV}$, and total and deal start time of $200 \mathrm{~ns}$ and $20 \mathrm{~ns}$, respectively. Figure 2 represents the two dimensional spectrum of the tail electron equivalent energy (Etail) divided by Eee versus Eee measured with a ${ }^{252} \mathrm{Cf}$ neutron fission source. The upper branch is from neutron interactions and the lower one is from gamma interaction since both neutrons and gamma's were emitted from the ${ }^{252} \mathrm{Cf}$ source. The neutrons and gamma's are clearly separated above $150 \mathrm{keV}$ Eee as shown in Fig. 2. The data from lower branch are also from the ${ }^{137} \mathrm{Cs}$

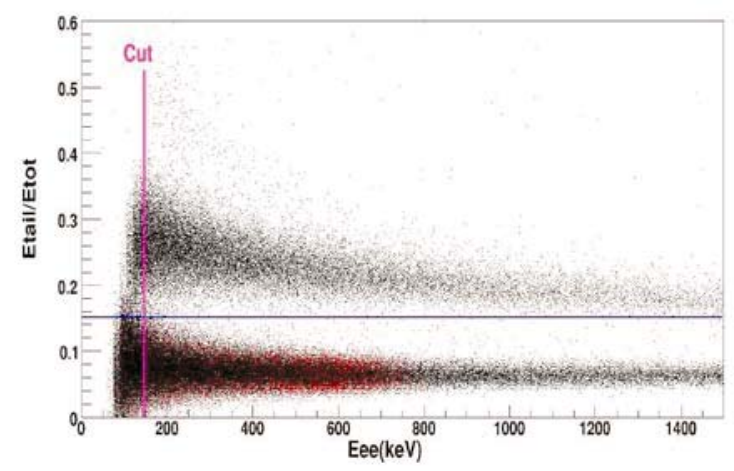

Fig. 2 The Etail/Eee vs Eee distribution measured with ${ }^{252} \mathrm{Cf}$ neutron source.

radioactive $\gamma$-ray irradiation and it proved that the lower branch is indeed only from gammas.

We can clearly separate neutron from gamma with the Etail/Eee $>0.15$ as shown in Fig 2. This value is used for tagging the neutron, and the trigger output is put into the NTM input for self triggering purpose. If there is the other detector which needs the neutron trigger information, a TTL output signal from NTM module can be applied.

\section{Flux and Dose monitoring at Proton Beam Line}

The BC523A detector was located at PEFP beam line of the MC-50 cyclotron facility to understand the activation of the nano-particles and effect to the nano-particle production. In the first setup, the neutron 
detector was located at $45^{\circ}$ with the distance of $40 \mathrm{~cm}$ from the beam exit window with a $5 \mathrm{~cm}$ lead block. In the other setup, the neutron detector was located just behind of a vial sample for nano-particle production study.

The followings were experimental setup for the test:

1) $40 \mathrm{~cm}, 45$ degree, $5 \mathrm{~cm} \mathrm{~Pb}$,

2) $40 \mathrm{~cm}$, behind, empty vial,

3) $40 \mathrm{~cm}$, behind, vial filled with water,

4) $40 \mathrm{~cm}$, behind, vial filled with water and nano-particles sources.

As described in the previous section, proton energy at the exit window of the proton beam line was $38 \mathrm{MeV}$. Proton beam current of $20 \mathrm{nA}$ was used for the test. It was

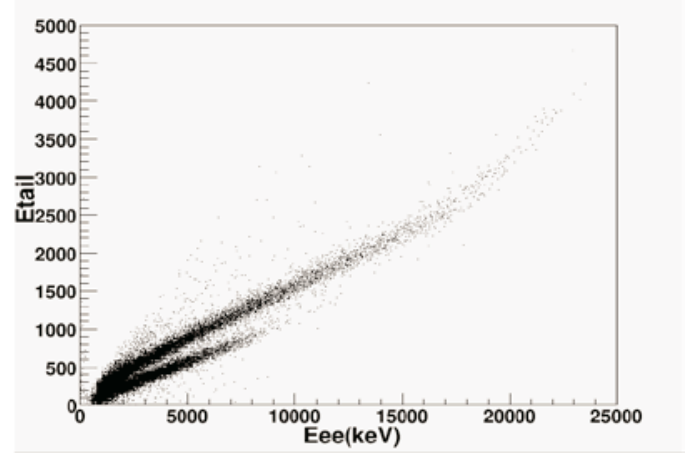

Fig. 3 Etail versus Eee distribution with the proton irradiation.

found that pile up effect became significant and $n / \gamma$ separation became difficult in case the beam current increased to $500 \mathrm{nA}$.

The BC523A liquid scintillator detector attached with a 2" PMT was used for the proton beam irradiation. It was attached directly to the window of the detector and the

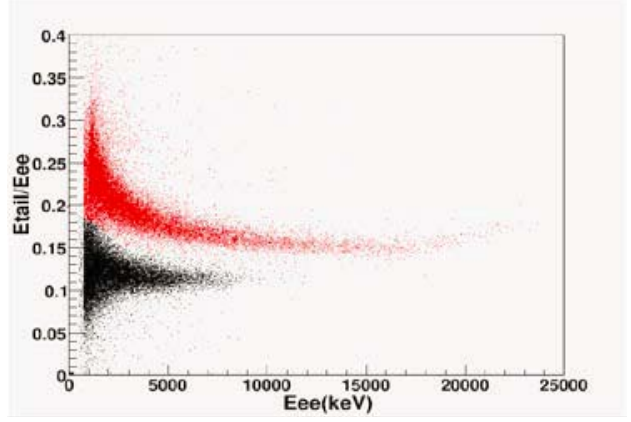

Fig. 4 The Etail/Eee versus Eee distribution with the proton irradiation. Upper and lower branch correspond to neutron and gamma, respectively.

high voltage was applied to the PMT. The PMT output is directly fed into the NTM module which was located inside of the proton beam line. Then, data acquisition was performed at the control room with a notebook computer through the Ethernet connection to the neutron tagger module directly. During the proton beam irradiation, it was found that energy spectra of neutron and gamma produced by the proton beam were much higher than the energy spectrum by the ${ }^{252} \mathrm{Cf}$ neutron source. Thus, high voltage of the PMT was lowered from $-1400 \mathrm{~V}$ to $-1100 \mathrm{~V}$ since saturation of the FADC in NTM caused non-linearity of the energy scale and neutron and gamma separation became worse. Recalibration of the Eee scale was done with a ${ }^{137} \mathrm{Cs}$ radioactive source.

Figure 3 shows the total (Eee) versus tail energy (Etail) spectra in the neutron detector by the proton irradiation and Fig. 4 shows Eee versus Etail/Eee spectrum. It was shown that the both neutron recoil and gamma energy are higher than the energy spectrum of ${ }^{252} \mathrm{Cf}$ neutron source as shown in Fig. 2. The energy threshold set by the software setting became $500 \mathrm{keV}$ in Eee, and neutron and gamma can be clearly separated above the energy threshold as shown in Fig. 4. The upper and lower band corresponds to neutron recoils and gamma's, respectively. Also the figure shows

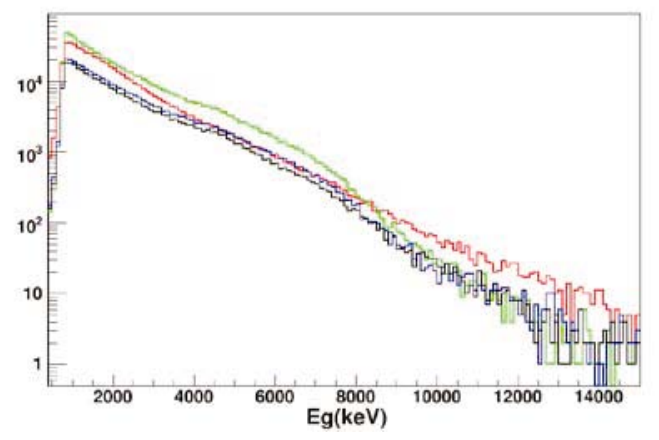

Fig. 5 Gamma energy spectra. Setup 1-4) corresponds to Green, Red, Blue and Black color, respectively.

that the neutron recoil energy spectrum is harder than gamma energy spectrum. Neutrons and gammas were separated by the proper selection criteria. Figure 5 shows the gamma energy deposition spectra of four different setups. As expected, setup 1) shows the softest energy spectrum since 5

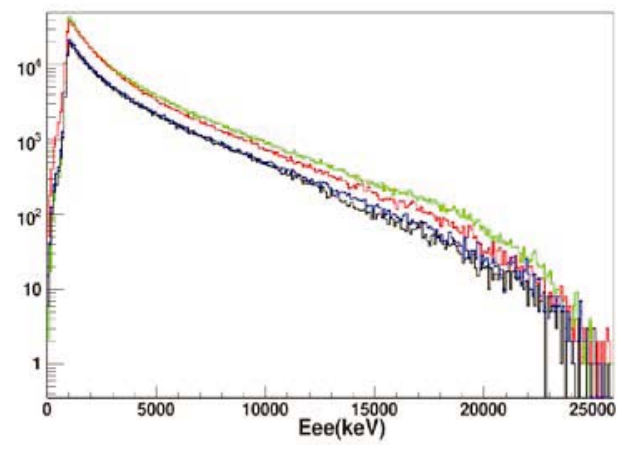

Fig. 6 Neutron recoiled Eee distribution.

$\mathrm{cm}$ of lead block was located in front of the BC523A detector. The end point of the gamma energy spectra is about $15 \mathrm{MeV}$.

Figure 6 shows the neutron recoiled Eee distribution. The 


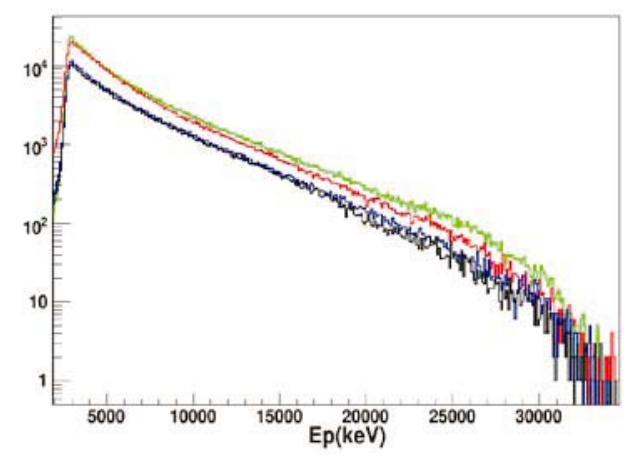

Fig. 7 Neutron recoiled proton energy distribution.

nonlinear response of scintillation light by the recoiled proton in a liquid scintillator is well known ${ }^{10)}$ and energy of the recoiled proton could be calculated from the Eee. Figure 7 shows the neutron recoiled proton energy spectra and the end point is approximately $35 \mathrm{MeV}$ which is close to the incident proton energy to the sample. Setups 2-4) shows that there are not much different energy distribution even if

Table 1 Flux, average energy and absorbed dose for both neutron and $\gamma$.

\begin{tabular}{|l|l|l|l|l|l|l|}
\hline setup & $\begin{array}{l}\text { n flux } \\
(\mathrm{n} / \mathrm{sec})\end{array}$ & $\begin{array}{c}\gamma \text { flux } \\
(\mathrm{n} / \mathrm{sec})\end{array}$ & $\begin{array}{c}\text { En } \\
(\mathrm{keV})\end{array}$ & $\begin{array}{c}\mathrm{E} \gamma \\
(\mathrm{keV})\end{array}$ & $\begin{array}{l}\text { n Dose } \\
(\mu \mathrm{Gy} / \mathrm{s})\end{array}$ & $\begin{array}{l}\gamma \text { Dose } \\
(\mu \mathrm{Gy} / \mathrm{s})\end{array}$ \\
\hline 2 & 64,000 & 53,000 & 6,114 & 2,054 & 0.71 & 0.20 \\
\hline 3 & 38,000 & 35,000 & 6,421 & 2,295 & 0.38 & 0.13 \\
\hline 4 & 35,000 & 29,000 & 6,407 & 2,252 & 0.34 & 0.12 \\
\hline
\end{tabular}

both neutron and gamma flux is higher for the setup 2). The counter information produced by the NTM module can be used for both neutron and gamma flux monitoring above threshold. Absorbed dose in the BC523A detector by neutron and gamma were calculated by using flux and energy information and the unit of the absorbed dose rate was denoted by $\mu \mathrm{Gy} / \mathrm{s}$. Since the density and atomic number of the BC523A detector is not much different from water, one can easily calculate absorbed dose in the water of the vial using absorbed dose in the BC523A detector. Table 1 shows the flux, average deposit energy and absorbed dose information of both neutron and gamma in the setup 2-4). Both gamma and neutron flux, absorbed dose is higher while average energy is lower for the empty vial than water filled vial as expected.

\section{Conclusion}

We measured the neutron and gamma flux, energy spectra and absorbed dose when nano-particle samples were irradiated with the proton beam. For the neutron and gamma separation, a cylindrical shape of BC523A liquid scintillator with the dimension of $\Phi 50 \mathrm{~mm} \times 50 \mathrm{~mm}$ was used and recently developed neutron tagger module was used for the signal digitization which has a pulse shape discrimination functionality. A ${ }^{137} \mathrm{Cs} \quad \gamma$-rays source and ${ }^{252} \mathrm{Cf}$ neutron fission source were used for the electron equivalent energy calibration and neutron and gamma separation optimization, respectively. The optimized study showed that neutrons and gamma's are clearly separated above $150 \mathrm{keV}$ in Eee.

Samples for nano-particle production were irradiated with $38-\mathrm{MeV}$ proton beam energy and proton beam current of 20 $\mathrm{nA}$ by the MC-50 cyclotron at the Korea Institute of Radiological and Medical Sciences. The fast neutron detector was located just behind the sample. The measured neutron and gamma flux, neutron and gamma induced radiation dose and energy spectra in the BC523A liquid scintillator were evaluated and compared with different sample setups.

This study showed that the developed neutron detection system is useful for the dose monitoring as well as flux monitoring of both neutron and gamma. The monitoring results can be applied for understanding of the activation of irradiated samples and the effect to the nano-particle production. Unfolding is necessary for neutron energy spectrum and it will be studied.

\section{Acknowledgment}

This work was supported by the user program of the Proton Engineering Frontier Project.

\section{References}

1) J. B. Birks, Theory and practice of scintillation counter, Pergamon press, Oxford (1964).

2) G. F. Knoll, Radiation Detection and Measurement, John Willey and Sons, New York (1999).

3) H.J. Kim et al., "Measurement of the neutron flux in the CPL underground laboratory and simulation studies of neutron shielding for WIMP searches", Astro. Part. Phys. 20, 549 (2004).

4) Yeong-Joon Kim, Gyoujin Cho and Jae Hee Song, "Ag+ concentration effect on the shape of $\mathrm{Au}$ nanomaterials under proton beam irradiation”, Nucl. Instr. Meth. A389, 73 (2007).

5) Jae Hee Song and Jong Ryoung Park, "Synthesis of Au and Ag Nanomaterials via Proton Beam Irradiation", J. of Korean Phys. Soc. 54 (5) 2143 (2009).

6) H. J. Kim, Eun-Joo Kim, S. Y. Kim, "Development of a Neutron Tagger Module using a Digital Pulse Shape Discrimination Method", N42-7, The 2008 IEEE Nuclear Science Symposium, Dresden, (2008).

7) F. Arneodo et al., "Calibration fo BC501A liquid scintillator cells with monochromatic neutron beams", Nucl. Instr. Meth. A418, 285 (1998).

8) R. Brunn and F. Rademakers, "ROOT-An Object Oriented Data Analysis Framework", Nucl. Instr. Meth. A389, 81 (1997).

9) K. R. Kim et al., Proceedings of the Agricultural Policy Advisory Committee, Gyeongju, Korea (2004).

10) R. A. Cecil, B. D. Anderson, R. Madey, Nucl. Instr. Meth. 161 43 (1979). 